\title{
A STUDY ON STAT6 EXPRESSION IN SOLITARY FIBROUS TUMOUR
}

\author{
Krishna Govindan'1, Nithya Jayakumar ${ }^{2}$
}

1 Professor and HOD, Department of Pathology, Government Medical College, Trivandrum, Kerala, India.

2Senior Resident, Department of Pathology, Government Medical College, Trivandrum, Kerala, India.

\section{BACKGROUND}

ABSTRACT

Solitary Fibrous Tumour (SFT) is an uncommon mesenchymal neoplasm of fibroblastic type arising anywhere in the body. Classic SFTs show neoplastic cells arranged in a pattern of less architecture with alternating hypo and hypercellular areas and prominent branching vasculature. Traditionally, CD-34 expression is the most consistent finding in SFTs, however this expression is also common in other tumours that may mimic SFT. Recently, a recurrent gene fusion NAB2-STAT6 has been identified as molecular hallmark of SFT. Molecular detection of the fusion gene and immunohistochemical expression of nuclear STAT6 can be helpful in diagnosing SFT.

Aim- To study the expression of STAT6 in SFT.

\section{MATERIALS AND METHODS}

23 cases of SFT diagnosed during a 2-year period. STAT6 immunohistochemistry was performed on 18 SFT cases and also on 8 possible mimickers of SFT, which were taken as the (negative) control.

\section{RESULTS}

Nuclear STAT6 positivity was present in 18/18 (100\%) cases of SFT, which were usually diffuse (4+ in 16 cases, $3+$ in 1 case and $1+$ in 1 case and intensity was strong in 15 cases, moderate in 2 cases and weak in 1 case). All other tumour types tested were negative for STAT6. CD-34 was done in all cases at initial diagnosis. 16/18 cases (88\%) were CD-34 positive. 2 cases (12\%) which were CD-34 negative were STAT6 positive. CD-99 and Bcl-2 were positive in most of the cases.

\section{CONCLUSION}

Strong nuclear STAT6 expression is largely specific for SFT and is helpful in distinguishing it from histologic mimics.

\section{KEY WORDS}

SFT, Solitary Fibrous Tumour, STAT6.

HOW TO CITE THIS ARTICLE: Govindan K, Jayakumar N. A study on STAT6 expression in solitary fibrous tumour. J. Evolution Med. Dent. Sci. 2018;7(45):4878-4883, DOI: 10.14260/jemds/2018/1086

\section{BACKGROUND}

This descriptive study of 'SFT was originally described in 1931 ' as a pleural tumour termed 'haemangiopericytoma,' but later it has been recognised that SFTs may occur in a wide range of anatomical locations.[1] Of extrapleural tumours, $40 \%$ arise in subcutaneous tissue with the remaining cases occurring in deep soft tissues, retroperitoneum, mediastinum, abdominal cavity and meninges. In 2013 edition of the World Health Organization classification of tumours of soft tissue and bone, the term of "haemangiopericytoma" has been made obsolete and the new classification no longer distinguishes between SFTs and HPCs.[2] Emerging together in all other sites except CNS, these tumours are categorised as SFTs under the classification of fibroblastic/ myofibroblastic tumours.[3] However, in CNS the grading is somewhat different. Classically, these tumours show a patternless proliferation of spindly tumour cells with alternating hypo and hypercellular areas, varying fibrosclerotic-to-myxoid

'Financial or Other Competing Interest': None.

Submission 27-08-2018, Peer Review 18-10-2018,

Acceptance 25-10-2018, Published 05-11-2018.

Corresponding Author:

Dr. Nithya Jayakumar,

Gopala, PRA 151 (C),

Padayani Road, Maruthankuzhy,

Vattiyoorkavu, P. O. Trivandrum-695013,

Kerala, India.

E-mail: nithyajk123@gmail.com

DOI: $10.14260 /$ jemds/2018/1086 stroma and staghorn vasculature with or without perivascular hyalinisation.[3] Other patterns observed are sheets, fascicles, herringbone, storiform and perivascular aggregation. Once considered to be distinct entities, now haemangiopericytoma considered to represent a cellular variant within the spectrum of solitary fibrous tumour, especially in the CNS where the terminology SFT/ HPC is retained.[4] Adults are most commonly affected. Although, most SFTs/ HPCs are benign, approximately $13-23 \%$ of tumours have been reported to be malignant.[5] Several histopathological criteria have been reported to be useful for determining malignancy in solitary fibrous tumour. These include increased tumour size, mitotic count, cellularity, presence of haemorrhage/ necrosis and nuclear pleomorphism.[6]

CD-34, CD-99 and B-cell lymphoma 2 (Bcl-2) were the three most useful positive immunohistochemical markers for diagnosing SFTs/ HPCs previously. However, their expressions are non-specific and also common in other tumours that mimic SFTs/ HPCs. Until recently, in 2013, three groups have discovered that NGFI-A binding protein-2 (NAB2) signal transducer and activator of transcription 6 (STAT6) fusion gene as a hallmark of SFTs/ HPCs by using wholeexome and transcriptome sequencing.[7] Schweizer et al recently demonstrated NAB2-STAT6 fusion gene could be rapidly detected by STAT6 immunohistochemistry, which exhibited strong nuclear STAT6 expression. ${ }^{[8]}$ Our aim was to study the STAT6 protein expression by immunohistochemistry in SFTs. We also reviewed the 
histopathology slides and other immunohistochemical markers, which were done previously in those cases like CD34, Bcl2 and CD-99.

\section{MATERIALS AND METHODS}

This is a descriptive study of 23 cases of solitary fibrous tumour diagnosed during a two years period in the Department of Pathology, Government Medical College, Thiruvananthapuram were included in the study. Of the 23 cases, we could retrieve the blocks of only 18 cases and STAT6 immunohistochemistry was performed on these. As (negative) control samples, some possible mimickers of SFT were stained. (1 synovial sarcoma, 1 DFSP, 1 Schwannoma, 1 neurofibroma, 1 MPNST, 1 Fibromatoses, 1 spindle cell lipoma, 1 fibrous histiocytoma).

The clinicopathologic parameters like age, location, gender, histopathology and immunohistochemical expression of STAT6 compared with other markers like CD-34, CD-99 and $\mathrm{Bcl} 2$ were assessed. Cases were scored positive for STAT 6 based on staining intensity ( 0 - no staining, 1 - weak, 2 moderate, 3- strong) and percentage of tumour cells $(0 \rightarrow<5 \%, 1+\rightarrow 5-25 \%, 2+\rightarrow 26-50 \%, 3+\rightarrow 51-75 \% ; 4+\rightarrow>75-$ $100 \%$ ) were recorded for each case with only nuclear staining considered positive.[9] CD-34, CD-99 and $\mathrm{Bcl} 2$ staining was performed at initial diagnosis and were considered positive when at least $10 \%$ of tumour cells showed strong staining. Negative controls were stained similarly.

Tissue Samples and Immunohistochemistry: - The tissue was fixed in $10 \%$ buffered formalin, routinely processed, embedded in paraffin; $4 \mu \mathrm{m}$ thick sections were stained with haematoxylin and eosin and immunohistochemistry with STAT6 was performed on the unstained sections using the commercially available antibody.

(Anti-STAT6 (Ab-645) antibody produced in rabbit; Sigma-Aldrich Chemical Pvt. Limited) in 1: 100 dilution on the test and control samples.

\section{RESULTS}

\section{Clinical Data}

23 patients were selected, which included 16 males (69\%) and 7 females (31\%) with an age range of 6 to 85 years. Majority belonged to the 40 - 60 years' age group (mean age was 53 years).

\section{Location}

We received cases from various sites, among which meninges was the most common followed by retroperitoneum, thigh (superficial plane) and maxillary sinus. Other sites included chest wall, abdominal wall, intra-abdomen, mediastinum, nasal cavity, tongue, cheek, paraspinal and pleura.

\section{Microscopic Findings}

Histologically, most of the cases showed typical features of SFT with patternless architecture of alternating hypo and hypercellular areas of spindle shaped cells and prominent staghorn vessels with some showing hyalinised walls (Figure 1). Out of 5 cases from the meninges, 2 showed a prominent storiform pattern with increased cellularity (Haemangiopericytoma phenotype) and increased mitosis (3-4/10 hpf) and hence were labelled as Grade 2 SFT/ HPC, which according to the 2016 WHO classification of CNS tumours is considered as malignant (Table 1) (Figure 2). Mitotic indexes have been reported in the range of 1 to 5 (mean 1.5) per $10 \mathrm{hpf}$ in the non-CNS sites.[10] The nuclei were spindled, ovoid and relatively uniform with some showing mild nuclear atypia. 2 cases diagnosed as malignant SFT in the non-CNS sites showed high cellularity, nuclear pleomorphism and mitosis $>4 / 10 \mathrm{hpf}$. The combination of high cellularity, higher mitotic indexes, conspicuous cellular pleomorphism and necrosis should most likely be considered as indicative of malignant potential. Prominent myxoid change was observed in one of the cases. There were 3 cases of lipomatous variant of SFT (Figure 3) with a differential diagnosis of well-differentiated liposarcoma for one case for which MDM2 gene study was advised.

\section{Immunohistochemical Findings (Table 2)}

STAT6 immunohistochemistry was performed on $18 / 23$ cases. Nuclear STAT6 positivity was present in 18/18 (100\%) cases of SFT, which were usually diffuse (4+ in 16 cases, $3+$ in 1 case and $1+$ in 1 case) and intensity was strong in 15 cases, moderate in 2 cases and weak in 1 case (Figure 4).

CD-34 was done in 22/23 cases at initial diagnosis. 20/22 cases were CD-34 positive. In the 18 cases where STAT6 was done, CD-34 was positive in 16/18 cases (88\%). 2 cases $(12 \%)$, which were CD-34 negative showed strong nuclear STAT6 positivity. CD-99 was done in 16/18 cases, where STAT6 was done at initial diagnosis. All cases were positive (100\%); however, 4 out of 16 cases showed only focal positivity. Bcl2 was also done in 16 of the 18 cases, of which 15 cases were positive (93\%) and one was negative. 4 out of the 5 showed only weak focal positivity (Table 2).

Figure 1

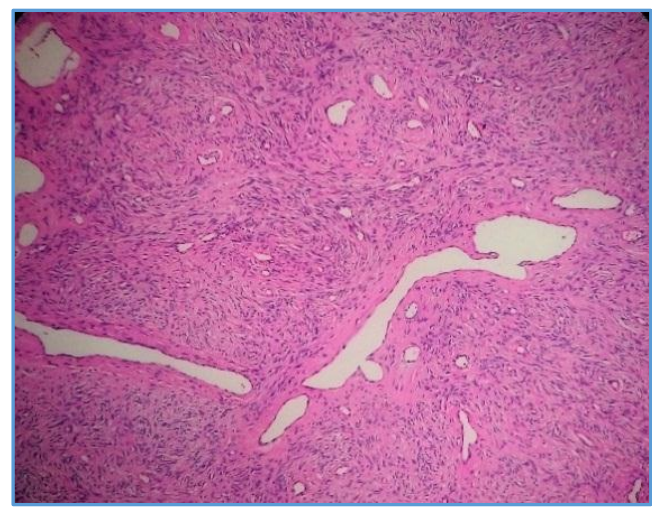

Figure 1(a). A Classic Histopathology of SFT showing Proliferated Spindly Cells arranged haphazardly with Stag Horn Vessels

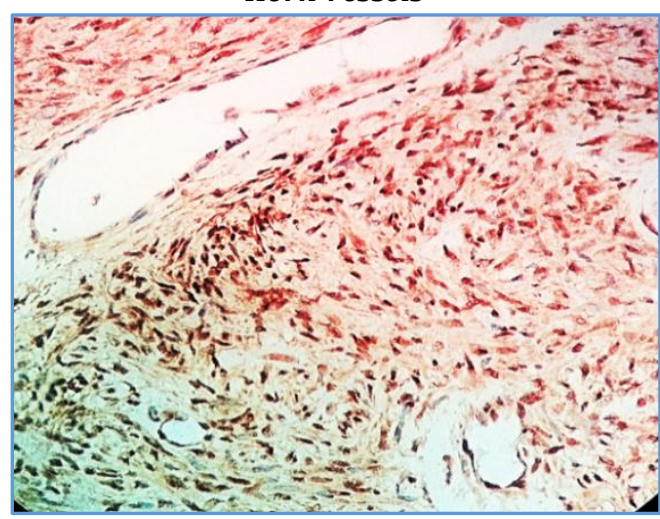

Figure 1(b). STAT6 showing Strong Nuclear Positivity 
Figure 2. A Case of Meningeal SFT/HPC, Intermediate Grade (Grade 2)

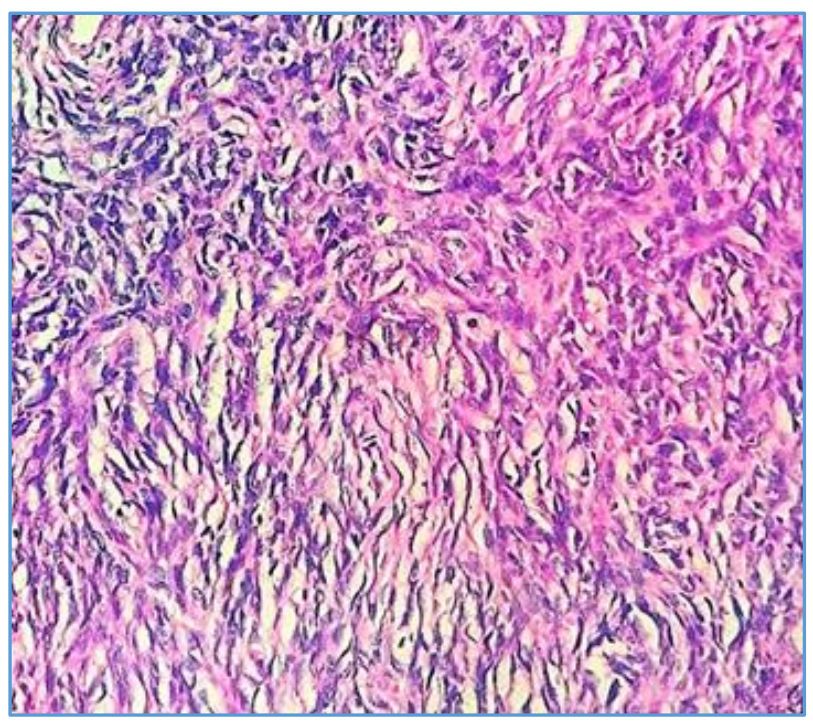

Figure 2(a). Histopathology shows a Cellular Neoplasm composed of Spindly Cells arranged predominantly in

Storiform Pattern with occasional Mitotic Figures

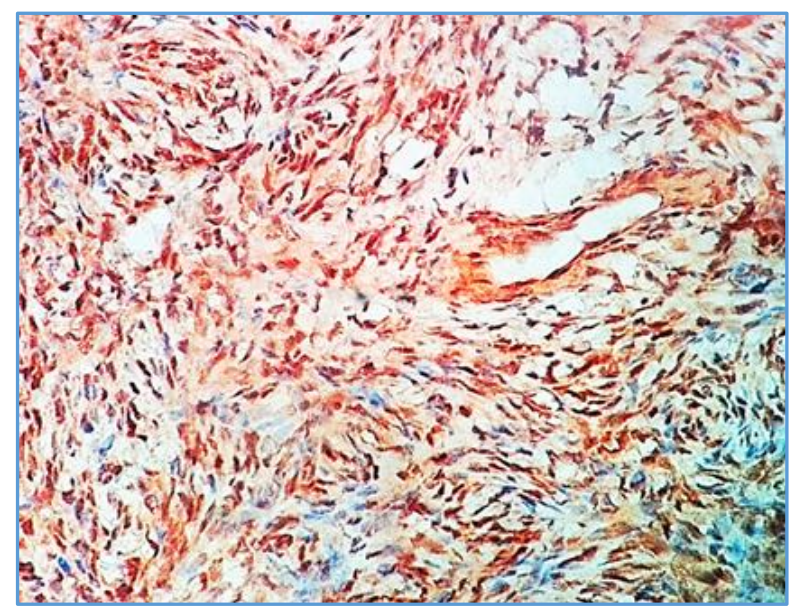

Figure 2(b). STAT6 showing Strong Nuclear Positivity in the Tumour Cells

Figure 3. Gross and Microscopy of a case of Lipomatous variant of SFT (Retroperitoneal).

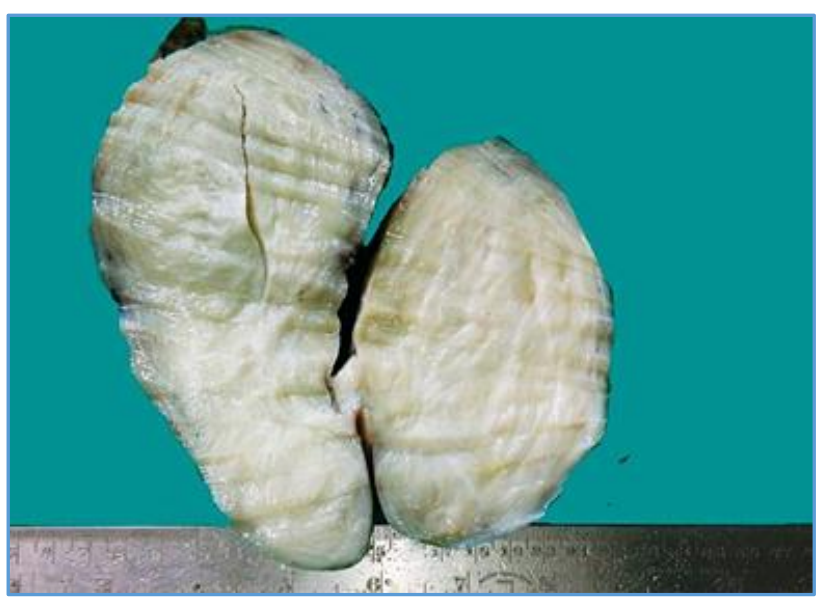

Figure 3(a). Gross photo showing a Solid Mass, Cut surface of which is Firm and Grey White

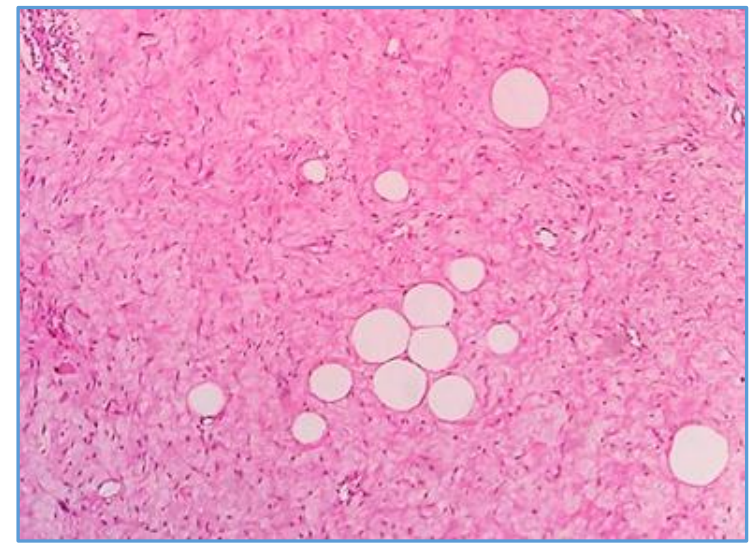

Figure 3(b). Microscopy showing Spindly Cells separated by Abundant Collagen with Clusters of Mature Adipocytes in Between

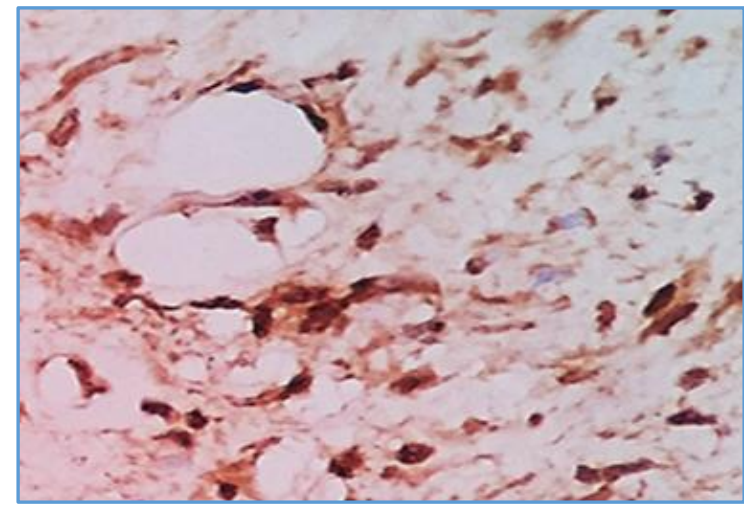

Figure 3(c). STAT6 showing Nuclear Positivity

Figure 4. STAT6 - Proportion and Intensity of Staining

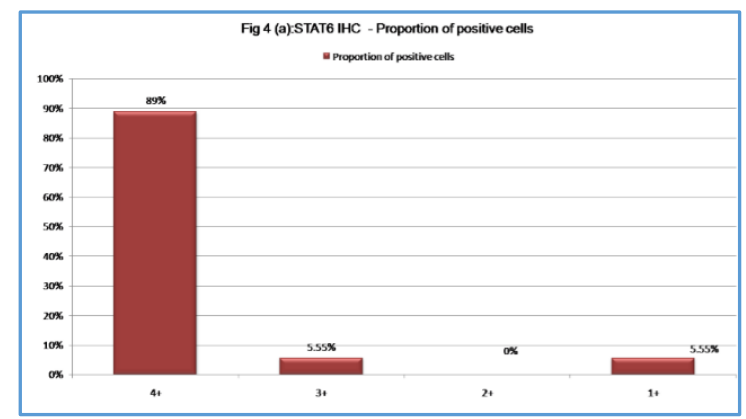

Figure 4(a). STAT6 IHC - Proportion of Positive Cells

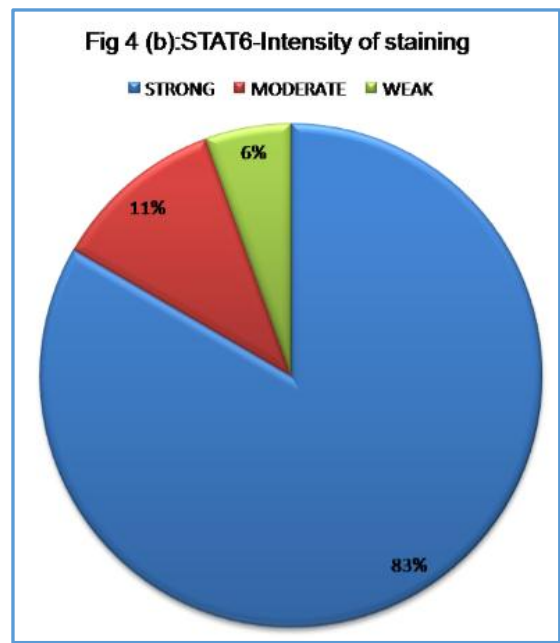

Figure 4(b). STAT6 - Intensity of Staining 


\begin{tabular}{|c|c|c|}
\hline \multicolumn{2}{|c|}{ CNS (WHO 2016) ${ }^{[\mathbf{1 3 ]}}$} & Non-CNS Sites[ ${ }^{[\mathbf{6}]}$ \\
\hline Grading & Benign $\rightarrow$ Surgical resection alone & Increased cellularity \\
\hline Grade 1 & Malignant (Less than 5 mitosis/ 10 High power field $\rightarrow$ Adjuvant \\
Radiotherapy & Pleomorphism \\
\hline Grade 2 & $\begin{array}{c}\text { Malignant } \\
\text { Grade 3 }\end{array}$ & $\begin{array}{c}\text { Mitoses (More than 4/10 High } \\
\text { Power Field) }\end{array}$ \\
\hline \multicolumn{2}{|c|}{$\begin{array}{c}\text { Table 1. Table Showing Criteria for Malignancy in SFTs[6, 13] } \\
\text { wHo Classification of Tumours of the Central Nervous System (Revised 4th Edition) }\end{array}$} \\
\hline
\end{tabular}

\begin{tabular}{|c|c|c|c|c|}
\hline Location & $\begin{array}{c}\text { STAT6 } \\
(n=18 / 23)\end{array}$ & $\begin{array}{c}\text { CD-34 } \\
(n=22 / 23)\end{array}$ & $\begin{array}{c}\text { CD-99 } \\
(n=16 / 23)\end{array}$ & $\begin{array}{c}\text { BCL2 } \\
(n=16 / 23)\end{array}$ \\
\hline Meninges (no: of cases 5) & $5 / 5$ & $5 / 5$ & $5 / 5$ & $5 / 5$ \\
\hline Maxillary sinus (no: of cases 2) & $2 / 2$ [1/2- weak focal positivity] & $2 / 2$ & $2 / 2$ & $1 / 1$ \\
\hline Pleura (no: of cases 2) & $2 / 2$ & $2 / 2$ & 1/1[focal+] & $1 / 1$ [focal+] \\
\hline Thigh (no: of cases 2) & $1 / 1$ & $2 / 2$ & $1 / 1$ & $2 / 2$ [1/2- focal+] \\
\hline Intra-abdomen (no: of cases 1) & - & $1 / 1$ & $1 / 1$ & $1 / 1$ \\
\hline Chest wall (no: of cases 1) & $1 / 1$ & $1 / 1$ & - & - \\
\hline Abdominal wall (no: of cases 1) & - & $1 / 1$ & $1 / 1$ & - \\
\hline Nasal mass (no: of cases 1) & $1 / 1$ & $0 / 1$ [negative] & $1 / 1$ & $0 / 1$ [negative] \\
\hline Cheek (no: of cases 1) & $1 / 1$ & $1 / 1$ & $1 / 1[$ focal +] & $1 / 1[$ focal+] \\
\hline Tongue (no: of cases 1) & - & $1 / 1$ & - & - \\
\hline Retroperitoneum (no: of cases 1) & $1 / 1$ & $1 / 1$ & $1 / 1[$ focal +] & $1 / 1$ \\
\hline Upper extremity (no: of cases 1) & $1 / 1$ & $0 / 1$ [negative] & - & - \\
\hline Scalp (no: of cases 1) & - & $1 / 1$ & - & $1 / 1$ \\
\hline Paraspinal (no: of cases 1) & $1 / 1$ & $1 / 1$ & - & - \\
\hline Mesentery (no: of cases 1) & $1 / 1$ & $1 / 1$ & $1 / 1[$ focal+] & $1 / 1[$ focal+] \\
\hline Mediastinum (no: of cases 1) & $1 / 1$ & - & $1 / 1$ & $1 / 1$ \\
\hline
\end{tabular}

( $\mathrm{N}=$ No: of cases where IHCs were performed out of the total 23 cases)

\section{DISCUSSION}

Solitary fibrous tumours belong to the fibroblastic group of neoplasms and they show variability in their clinical behaviour.[11] Initially described in pleura, they were later observed to occur in wide range of anatomical locations. SFTs/ HPCs have recurrently displayed a paracentric inversion in chromosome $12 \mathrm{q} 13$, forming a fusion of two neighbouring and partly overlapping genes: NAB2 which is transcribed from centromere to telomere and STAT6 which is transcribed from telomere to centromere. Recently, researchers have identified this novel NAB2-STAT6 fusion gene that represents the first molecular feature unique to SFTs/ HPCs of any anatomical sites and regardless of benign or malignant.[12] Demonstration by molecular methods is expensive and hence they are not used extensively in many laboratories. Also as these genes are located in close proximity on $12 q 13$, this fusion can only rarely be detected by conventional chromosomal banding or fluorescence in situ hybridisation analysis.[11] Schweizer et al demonstrated that NAB2-STAT6 fusion gene can be detected by STAT6 immunohistochemistry, which gives a nuclear positivity.[8] Although, most SFTs pursue a benign course, recurrence or metastasis develops in $5-10 \%$ of cases. The criteria for malignancy in SFT is non-CNS sites that include increased cellularity, mitoses, nuclear pleomorphism and necrosis. However, in the CNS SFT/ HPCs are graded into 1, 2, 3, of which grade 1 is benign. Grade 2 and 3 are malignant and is based on the number of mitotic figures $(<5 / 10$ high power field is grade 2 and $>5 / 10$ high power field is grade 3 ). (Table 16,13])

In a large study conducted by Demico et al, they studied the expression of STAT6 in 2021 mesenchymal tumours, including 240 SFTs. Strong nuclear STAT6 positivity was seen in 285 of 2021 tumours including 206 of 240 SFTs. Although their sensitivity was only $87 \%$, further analysis revealed that among cases resected within the past 5 years, STAT6 immunohistochemistry displayed $97 \%$ sensitivity for solitary fibrous tumours.[14] Demico et al also demonstrated that 49/408 well/de-differentiated liposarcomas, 8/65 unclassified sarcomas and 14/184 desmoids were also positive for STAT6; however, showing both cytoplasmic and nuclear positivity. Expression in SFT was mainly limited to the nucleus.[14]

In the study conducted by Doyle et al nuclear expression of STAT6 was observed in 59/60 (98\%) cases, which was usually diffuse and intense. All other tumour types were negative for STAT6, except for three dedifferentiated liposarcomas and one deep fibrous histiocytoma, which showed weak staining.

Few other studies also showed a $100 \%$ strong nuclear expression of the marker in solitary fibrous tumours.[15,16] Study conducted by Yoshida et al on 49 SFTs showed STAT6 expression in all the cases $(100 \%)$ that was restricted in the nucleus, mostly in a diffuse and strong manner, irrespective of the tumour sites and histologic patterns.[16] In their study, 
only 4 non-SFT tumours (2.5\%) exhibited weak nuclear STAT6 expression.

In our study, nuclear expression of STAT6 was present in all cases $(100 \%)$, which was diffuse in $89 \%$ cases and strong in $83 \%$ cases. This is equivalent to some of the previous studies.[15,16] One case showed weak positivity in terms of extent and intensity of staining, which may be attributed to uneven tissue fixation or loss of antigenicity in older cases. CD-34 showed strong positivity in this case. So the sensitivity can be increased when these markers are combined together. All other tumour types which can be the possible mimickers of SFT examined (i.e. one case each of synovial sarcoma, DFSP, schwannoma, neurofibroma, MPNST, fibromatosis, spindle cell lipoma, benign fibrous histiocytoma) were negative for STAT6 except for some non-specific cytoplasmic staining in few cases, but clearly lacking the nuclear expression. However, larger number of study samples can provide more insight to the staining of STAT6 in other mesenchymal tumours. Some studies show loss of expression of STAT6 in malignant solitary fibrous tumours.[17] However, we observed no difference in expression of the marker in the two malignant cases.

Doyle et al reported the expression of moderate-to-strong STAT 6 in upto $12 \%$ of de-differentiated liposarcomas and determined that STAT6 expression was due to gene locus inclusion in the 12q13 15 amplicon characteristic of this tumour.[11] Demicco et al also found similar rates of high nuclear STAT6 expression in both well-differentiated (14\%) and de-differentiated (8\%) liposarcomas in their study cohort. Both studies showed that nuclear expression of STAT6 was usually seen in conjunction with an equivalent or near-equivalent degree of cytoplasmic staining and their findings suggested a possible amplification and subsequent overexpression of full-length STAT6.[14] In our study, the lipomatous variant of SFT confused with well-differentiated liposarcoma showed nuclear STAT6 positivity alone, which was diffuse and strong without cytoplasmic staining, thus favouring a diagnosis of SFT rather than a well-differentiated liposarcoma. However, in such cases an MDM2 gene amplification study by immunohistochemistry or by molecular methods along with a nuclear and cytoplasmic STAT6 positivity can confirm or exclude this possibility.

CD-34 has been reportedly revealed to be diffusely and strongly expressed in many cases of SFTs/ HPCs. However, approximately 5 - 10\% of SFTs/ HPCs can be negative for CD34.[3] CD-34 is not entirely specific for SFTs/ HPCs, because it can be expressed in a variety of mesenchymal tumours. In the study conducted by Tai et al, STAT6 exhibited distinctive nuclear labelling in seven of eight CD34-negative solitary fibrous tumours with typical histology indicating its better sensitivity.[9] Our study also showed distinct nuclear positivity of STAT6 in the 2 cases, which were CD-34 negative. So it can be a good reliable marker in CD-34 negative solitary fibrous tumours.

Other markers that are variably expressed in SFT include CD-99 and Bcl2. However, these markers are positive in other soft tissue tumours that may mimic SFT. In our study, Bcl2 was expressed in 15 of 16 (93\%) cases with weak focal positivity in 4 cases. One case which was negative for $\mathrm{Bcl} 2$ was STAT6 positive.

CD-99 was positive in all the cases (100\%). However, as these two markers are neither specific nor sensitive, a more sensitive and specific marker like STAT6 when used in combination can aid in arriving at an accurate diagnosis. Also, in cases where both markers are positive, immunohistochemical marker like TLE-1 along with molecular confirmation by demonstrating SYT-SSX fusion could be done in these cases to rule out a Synovial sarcoma, which was not done in our study. However, demonstration of STAT6 in these tumours rules out the above possibility as synovial sarcomas are negative for STAT6 implying the lack of NAB2-STAT6 fusion. The limitation of our study was the study period and the number of cases studied. However, applying the marker routinely to diagnose and differentiate solitary fibrous tumour from other mesenchymal tumours can bring in more light into the utility of this marker and the problems encountered.

\section{CONCLUSION}

Our immunohistochemical study confirms the diagnostic sensitivity and specificity of STAT6 nuclear immunoexpression in the diagnosis of solitary fibrous tumours and in the differential diagnosis of histological mimics. Also, it is a good and reliable marker in CD-34 negative solitary fibrous tumours.

\section{REFERENCES}

[1] Fletcher CDM, Unni KK, Mertens F. World Health Organization classification of tumours. Pathology and Genetics of tumours of soft tissue and bone. Lyon: IARC Press 2002: p. 86-90.

[2] Han Y, Zhang Q, Yu X, et al. Immunohistochemical detection of STAT6, CD34, CD99 and BCL-2 for diagnosing solitary fibrous tumors/ hemangiopericytomas. Int J Clin Exp Pathol 2015;8(10):13166-75.

[3] Fletcher CDM, Bridge JA, JC L. Extrapleural solitary fibrous tumour. In: Fletcher CDM, Bridge JA, Hogendoorn PCW, et al. eds. World Health Organisation classification of tumours of soft tissue and bone. $4^{\text {th }}$ edn. Lyon: IARC Press 2013: p. 80-2.

[4] Demicco EG, Park MS, Araujo DM, et al. Solitary fibrous tumor: a clinicopathological study of 110 cases and proposed risk assessment model. Mod Pathol 2012;25(9):1298-306.

[5] Hanau CA, Miettinen M. Solitary fibrous tumor: histological and immunohistochemical spectrum of benign and malignant variants presenting at different sites. Hum Pathol 1995;26(4):440-9.

[6] Enzinger FM, Smith BH. Hemangiopericytoma. An analysis of 106 cases. Hum Pathol 1976;7(1):61-82.

[7] Mohajeri A, Tayebwa J, Collin A, et al. Comprehensive genetic analysis identifies a pathognomonic NAB2/STAT6 fusion gene, non-random secondary genomic imbalances, and a characteristic gene expression profile in solitary fibrous tumor. Genes Chromosomes Cancer 2013;52(10):873-86.

[8] Schweizer L, Koelsche C, Sahm F, et al. Meningeal hemangiopericytoma and solitary fibrous tumors carry the NAB2-STAT 6 fusion and can be diagnosed by nuclear expression of STAT6 protein. Acta Neuropathol 2013;125(5):651-8. 
[9] Tai HC, Chuang IC, Chen TC, et al. NAB2-STAT6 fusion types account for clinicopathological variations in solitary fibrous tumors. Mod Pathol 2015;28(10):1324-35.

[10] Fletcher CDM. Diagnostic histopathology of tumours. $4^{\text {th }}$ edn. Philadelphia: Elsevier Saunders 2013.

[11] Doyle LA, Vivero M, Fletcher CD, et al. Nuclear expression of STAT6 distinguishes solitary fibrous tumor from histologic mimics. Mod Pathol 2014;27(3):390-5.

[12] Chmielecki J, Crago AM, Rosenberg M, et al. Wholeexome sequencing identifies a recurrent NAB2-STAT6 fusion in solitary fibrous tumors. Nat Genet 2013;45(2):131-2.

[13] Louis DN, Ohgaki H, Wiestler OD, et al. WHO Classification of tumours of The Central Nervous System. Revised 4th edn. Lyon: IARC 2016.
[14] Demicco EG, Harms PW, Patel RM, et al. Extensive survey of STAT6 expression in a large series of mesenchymal tumors. Am J Clin Pathol 2015;143(5):672-82.

[15] Vogels RJ, Vlenterie M, Versleijen-Jonkers YM, et al. Solitary fibrous tumor - clinicopathologic, immunohistochemical and molecular analysis of 28 cases. Diagn Pathol 2014;9:224.

[16] Yoshida A, Tsuta $K$, Ohno $M$, et al. STAT6 immunohistochemistry is helpful in the diagnosis of solitary fibrous tumors. Am J Surg Pathol 2014;38(4):552-9.

[17] Wang C, Qi Y, Liu R, et al. Immunohistochemical evaluation of stem cell markers and signal transducer and activator of transcription 6 (STAT6) in solitary fibrous tumors. Int J Clin Exp Pathol 2015;8(9):1058594. 